\title{
An update on chronic lung allograft dysfunction
}

\author{
Sakhee Kotecha, Miranda A. Paraskeva, Kovi Levin, Gregory I. Snell \\ Lung Transplant Service, Alfred Hospital, Melbourne, Australia \\ Contributions: (I) Conception and design: S Kotecha, MA Paraskeva, GI Snell; (II) Administrative support: None; (III) Provision of study materials or \\ patients: None; (IV) Collection and assembly of data: None; (V) Data analysis and interpretation: None; (VI) Manuscript writing: All authors; (VII) \\ Final approval of manuscript: All authors. \\ Correspondence to: Dr. Sakhee Kotecha, MD. Lung Transplant Service, Alfred Hospital, Commercial Road, Melbourne, 3004, Victoria, Australia. \\ Email: s.kotecha@alfred.org.au.
}

\begin{abstract}
Chronic lung allograft dysfunction (CLAD) remains a significant challenge and the major determinant of morbidity and mortality post lung transplantation (LTx). The definition of CLAD has evolved significantly over the last ten years, reflecting better understanding of pathophysiology and different phenotypes. While there is an agreed consensus approach to CLAD, questions remain regarding the limitations of lung function parameters as well as the role of imaging and histopathology. Here we present a current snapshot of the definition of CLAD, its evolution and future directions.
\end{abstract}

Keywords: Lung transplantation (LTx); chronic lung allograft dysfunction (CLAD)

Submitted Oct 09, 2019. Accepted for publication Dec 06, 2019.

doi: 10.21037/atm.2020.01.05

View this article at: http://dx.doi.org/10.21037/atm.2020.01.05

\section{Introduction}

Since the inception of lung transplantation (LTx) more than 50 years ago, it has evolved significantly with improved surgical and medical management (1). Despite these substantial improvements, LTx still has the poorest survival for any solid organ transplant, with a median survival of 6 years in adults and 5.5 years in pediatric recipients (2). The major cause for morbidity and mortality post LTx is chronic lung allograft failure, the definition of which has evolved dramatically since it was first described three decades ago. Nevertheless, the incidence of this chronic rejection, historically termed bronchiolitis obliterans syndrome, increases with time post LTx, affecting $22 \%$ at 2 years, $50 \%$ at 5 years and $77 \%$ at 10 years post LTx (2). The advent of more sophisticated immunosuppression and antimicrobial prophylaxis strategies has improved outcomes but chronic lung allograft dysfunction (CLAD), the contemporary term coined for lung allograft failure, remains the major challenge of LTx. This chapter will highlight the evolution of this definition and expand the ongoing challenges of CLAD that require further study and attention.

\section{The evolution from obliterative bronchiolitis (OB) to CLAD}

The clinical problem of a chronically failing lung allograft was originally described in the late 80 's and was perceived as a form of chronic rejection and primarily considered as the development of histologic OB (3). Subsequently it became evident that the dysfunctional allograft could better be defined via the presence of obstructive spirometry, noting the pathologic description of $\mathrm{OB}$ required a confirmatory tissue diagnosis that was problematic to obtain and was only variably actually present on transbronchial biopsy histology (4). The term bronchiolitis obliterans syndrome (BOS) was thus coined and defined. Importantly, using spirometric cut-offs calculated relative to the best achieved previously post-LTx, it became possible to score the severity of the lung dysfunction. This definition primarily framed 


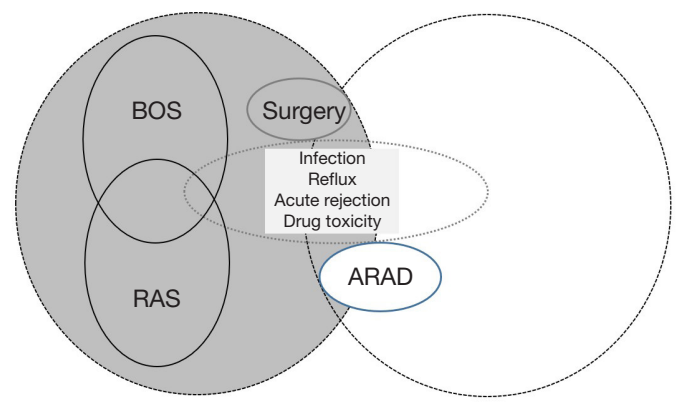

Chronic Lung Allograft Dysfunction Acute Lung Allograft Dysfunction

Figure 1 The state of lung allograft dysfunction circa 2016. BOS, bronchiolitis obliterans syndrome; RAS, restrictive allograft syndrome; ARAD, azithromycin-reversible allograft dysfunction.

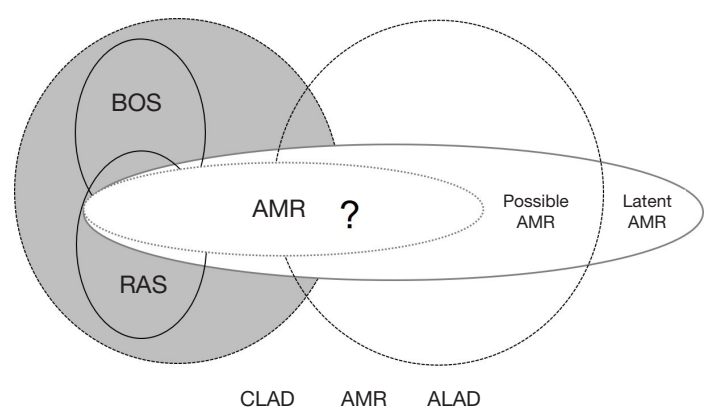

Figure 2 The position of lung allograft AMR in lung allograft dysfunction circa 2016. BOS, bronchiolitis obliterans syndrome; RAS, restrictive allograft syndrome; AMR, antibody mediated rejection; CLAD, chronic lung allograft dysfunction; ARAD, azithromycin-reversible allograft dysfunction.

BOS as an irreversible allo-immune process, but noted other innate immune /lung injury contributors to a nett physiological decline.

Recognizing the major clinical relevance of severe BOS as the most important descriptor of LTx morbidity and mortality has seen BOS as the target, or at least a major secondary endpoint, of many studies (5). Notwithstanding the broad benefit of considering BOS as a transplant outcome, its descriptors were shown to have limitations. The presence and even the actual date onset of BOS have actually proved challenging to exactly define. Confounding situations were noted and it became clear the date of onset was essentially best assessed some months later rather than in real time. In single LTx the definition struggled to take into account issues related to deterioration of the native lung. In other cases, post-pneumonic or postsurgical scarring, phrenic nerve palsy, aspiration or obesity could contribute to significant changes in lung function, sometimes reversing at least temporarily. In contrast to the original perception that BOS was describing chronic allograft rejection, it became clear that there were many common allograft events that were not pathogenic alloimmune processes $(5,6)$. The concept of BOS being irreversible was also challenged by reports showing that, at least temporarily, the macrolide antibiotic azithromycin could be shown to improve obstructive spirometric measures (7) and this was subsequently named azithromycin-reversible allograft dysfunction (ARAD).

Further, it also became evident that BOS as defined with an obstructive spirometric phenotype, was not able to describe all cases of lung dysfunction, as cases with a restrictive phenotype were also being seen (8) in association with radiographic infiltrates and this was eventually named restrictive allograft syndrome (RAS) (9). The evolution of Luminex testing for donor-specific anti- Human Leukocyte Antigen antibodies raised additional questions as to the overlap of these entities with acute and chronic antibody mediated lung rejection $(10,11)$. Figures 1,2 summarize the clinician's evolved view of lung dysfunction by the year 2016.

To reconcile all the above controversies, new data, strategies and discussions, an International Society for Heart and Lung Transplantation (ISHLT) CLAD Consensus Report was developed to update the field and create a new platform for further study. The use of the term CLAD was locked in as the umbrella term to describe the clinical expressions of a number of processes that lead to significant and persistent deterioration in the function of the lung allograft. It was defined as persistent, obstructive or restrictive or mixed according to spirometry (6) (Table 1). It excludes confounders such as ageing, surgical, mechanical, airway stenosis, post infectious scarring or rejection that clears in $<3$ months. It can also be reset beyond 6 months after surgery or mechanical acute changes and is summarized in Figure 3, which highlights the complexities and overlap of the potential aetiologies contributing of allograft dysfunction including those at baseline (baseline lung allograft dysfunction, BLAD) and those that develop within the first 3 months (Acute lung allograft dysfunction, ALAD). The severity was also scored spirometrically (Table 2). A simultaneously released ISHLT RAS Consensus Report (12) was also developed that defined this entity via restrictive spirometry, with a co-existent $10 \%$ decrease in 
Table 1 Current CLAD subtype classification features (6)

\begin{tabular}{llll}
\hline & Obstruction FEV $/$ FVC $<0.7$ & Restriction TLC $<90 \%$ baseline & HRCT opacities \\
\hline BOS & + & - & - \\
RAS & - & + & + \\
Mixed (change from one subtype to another) & + & + & + \\
Undefined (either type present) & + & + & + \\
& + & + & - \\
\hline
\end{tabular}

+, present; -, absent. CLAD, chronic lung allograft dysfunction; FEV1, forced expiratory volume in 1 second; FVC, forced vital capacity; TLC, total lung capacity; HRCT, high resolution computed tomography; BOS, bronchiolitis obliterans syndrome; RAS, restrictive allograft syndrome. Reprinted from Verleden GM, Glanville AR, Lease ED, et al. Chronic lung allograft dysfunction: Definition, diagnostic criteria, and approaches to treatment-A consensus report from the Pulmonary Council of the ISHLT. J Heart Lung Transplant 2019;38:493-503. May 2019, with permission from Elsevier.

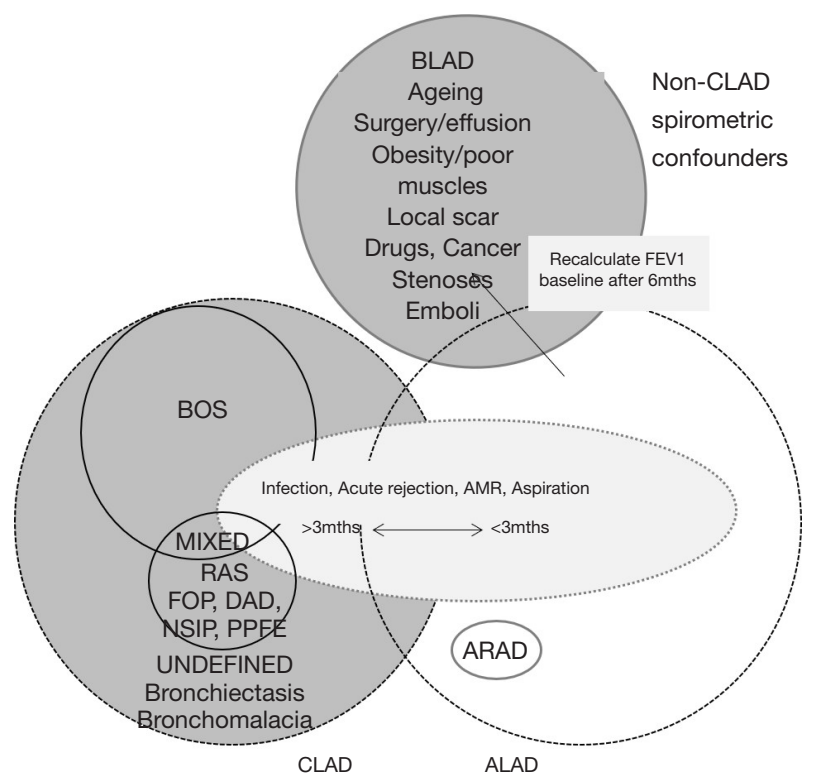

Figure 3 The current state of lung allograft dysfunction. BLAD, baseline allograft dysfunction; CLAD, chronic lung allograft dysfunction; $\mathrm{FEV}_{1}$, forced expiratory volume in 1 second; BOS, bronchiolitis obliterans syndrome; RAS, restrictive allograft syndrome; AMR, antibody mediated rejection; FOP, fibrinoid organising pneumonia; DAD, diffuse alveolar damage; NSIP, non-specific interstitial pneumonia; PPFE, pleuro-parenchymal fibro-elastosis; ALAD, acute lung allograft dysfunction. ARAD, azithromycin-reversible allograft dysfunction.

total lung capacity (TLC) and persistent parenchymal (and/ or) pleural radiographic infiltrates. Notably the Consensus group decided to score all CLAD spirometric phenotypes according to a single forced expiratory volume in $1 \mathrm{~s}\left(\mathrm{FEV}_{1}\right)$ based score (6).
The challenge of using these new definitions however, remains the heterogeneity of allograft dysfunction seen in the real world, as well as differences in availability of investigations. There are practical issues in routine follow up that will limit the application of these new definitions in both day-to-day management of the lung transplant recipient, but also in utilizing prior or current research protocols which have relied on the traditional retrospective definition of baseline spirometry and consequent CLAD.

\section{The definition of RAS and the limitations of body plethysmography}

The use of TLC decline as part of the diagnostic criteria to define RAS was first described by Sato et al. (9), who identified that $30 \%$ of their CLAD cohort presented with sustained impairment of $\mathrm{FEV}_{1}$ coupled with a declining TLC $\leq 90 \%$ of baseline. This group additionally identified specific radiological and histopathological features that demarcated RAS from BOS including upper zone infiltrates on imaging (Figure 4) and diffuse alveolar damage on histopathology $(9,13,14)$. Numerous other studies have built on and supported these findings confirming the impact of a $\geq 10 \%$ decline in TLC on survival (15-17).

Whilst the definition of the BOS variant of CLAD has remained essentially unchanged (i.e., a fall of $\mathrm{FEV}_{1}$ $\geq 20 \%$ from baseline with evidence of airflow limitation $\left[\mathrm{FEV}_{1}\right.$ /forced vital capacity $\left.(\mathrm{FVC})<0.7\right]$ and an absence of opacities on chest imaging), the consensus definition of RAS has evolved. RAS is currently defined as evidence of $\mathrm{FEV}_{1} \geq 20 \%$ decline from baseline; in addition to a decline of TLC to $\leq 90 \%$ of baseline; and evidence of persistent opacities on chest imaging (12). As such, TLC and body 
Table 2 Current CLAD severity spirometric scoring (6)

\begin{tabular}{ll}
\hline CLAD stage & Spirometric values \\
\hline CLAD 0 & Current FEV $_{1}>80 \%$ baseline \\
CLAD 1 & Current FEV $_{1} 66-80 \%$ baseline \\
CLAD 2 & Current FEV $_{1} 51-65 \%$ baseline \\
CLAD 3 & Current FEV $135-50 \%$ baseline \\
CLAD 4 & Current FEV $1<35 \%$ baseline \\
\hline
\end{tabular}

Baseline, average of previous best 2 figures $>3$ weeks apart. CLAD, chronic lung allograft dysfunction; FEV1, forced expiratory volume in 1 second. Reprinted from Verleden GM, Glanville AR, Lease ED, et al. Chronic lung allograft dysfunction: Definition, diagnostic criteria, and approaches to treatment-A consensus report from the Pulmonary Council of the ISHLT. J Heart Lung Transplant 2019;38:493-503. May 2019, with permission from Elsevier.

plethysmography have become a prerequisite in the diagnostic algorithm investigating CLAD. This is likely to be challenging on multiple fronts.

Firstly, whilst body plethysmography is the preferred technique in measuring TLC, it is not universally used at all lung transplant centers (12) and at some centers is not permitted in patients with pan-resistant or multidrug resistant organisms (17). Whilst the standardization of TLC measurement recommended by the consensus document at 3 and 6 months post-transplant (6) may assist the further characterization of CLAD for individual new transplant patients who can be monitored as recommended and followed as cohorts into the future, it limits the current capacity of institutions to examine CLAD in their current cohorts and to undertake retrospective cohort studies as the majority of lung transplant recipients have not undergone routine body plethysmography thus far.

Secondly, there are limitations in our understanding of the evolution of TLC following LTx. Unlike other indices of lung function ( $\mathrm{FEV}_{1}$ and $\mathrm{FVC}$ in particular) which have been extensively studied (18-20), little is known of the natural history of TLC and in particular the timing of its peak after transplantation. Baseline TLC has variably been defined as the mean of the two TLC measurements obtained at the time of the best two $\mathrm{FEV}_{1}$ measures (9) to the mean of the two best TLC values within 3 months of baseline $\mathrm{FEV}_{1}(17)$. The current consensus documents less specifically defines baseline TLC as: the mean of two TLC measurements taken at the time of or very near to the two best post-operative $\mathrm{FEV}_{1}$ measures (12). Whilst the consensus documents suggest baseline TLC measures be undertaken at 3 and 6 months (6), this timing is not aligned with our understanding of the attainment of baseline spirometry and in particular baseline $\mathrm{FEV}_{1}$ (i.e., peak $\mathrm{FEV}_{1}$ ) which has repeatedly been shown to occur at a mean of 9 months post-transplant $(18,20)$.

Thirdly, the studies that have supported the use of TLC in the diagnosis of RAS $(9,17,18)$ have extrapolated our understanding of standard respiratory physiology in normal subjects to lung transplant recipients stating that TLC is more accurate in distinguishing restrictive physiology than declines in FVC (21). Whilst this is likely to be true, the absence of detailed longitudinal studies examining lung volumes and spirometry post-transplant does mean the true correlation between TLC and FVC decline in CLAD is currently unknown.

\section{Alternate strategies for making RAS diagnosis}

Though not part of the consensus definition an increasing body of work has assessed alternate methodologies of diagnosing RAS or restrictive CLAD utilising the spirometric indices of lung function including $\mathrm{FEV}_{1} / \mathrm{FVC}$ ratio and $\mathrm{FVC}$, in conjunction with changes noted on chest imaging and in particular infiltrates noted on high resolution computed tomography (HRCT) scanning.

Todd et al. first highlighted that evidence of FVC decline to $<80 \%$ of baseline at CLAD onset influenced survival (19) and accounted for approximately $30 \%$ of CLAD in their cohort, a similar proportion to those reported as having RAS based on TLC decline in other studies $(9,16,17)$. In this cohort study, the majority of the transplant recipients with CLAD and a decline in FVC had concurrent evidence of CT opacities and this constellation of features was associated with poorer survival. This has been validated by a number of subsequent studies $(18,22)$ and supports the use of $\mathrm{FVC}$ and $\mathrm{FEV}_{1} / \mathrm{FVC}$ ratio in the absence of TLC to add prognostic significance to characterisation of CLAD. As previously mentioned, the absence of studies examining longitudinal changes in spirometry and lung volumes prevents a true understanding of the correlation between FVC and TLC decline in the definitions of CLAD. However, these studies support the idea that there is likely a significant overlap in CLAD phenotype in those with TLC decline and those with FVC decline, given the shared clinical characteristics of poorer survival, radiological changes and evidence of restriction on lung function (9,16-19). Notwithstanding the potential for decline in FVC to be 

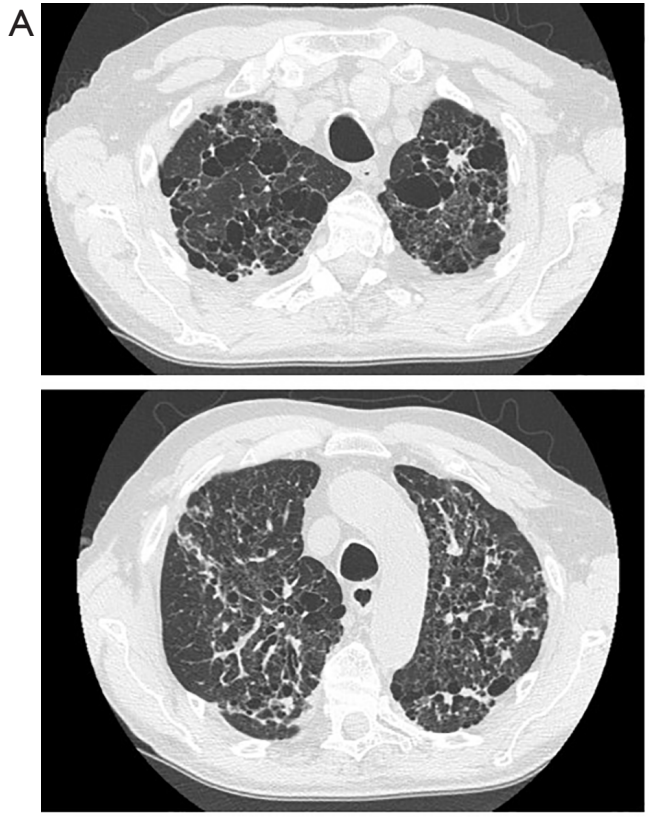

4a: RAS
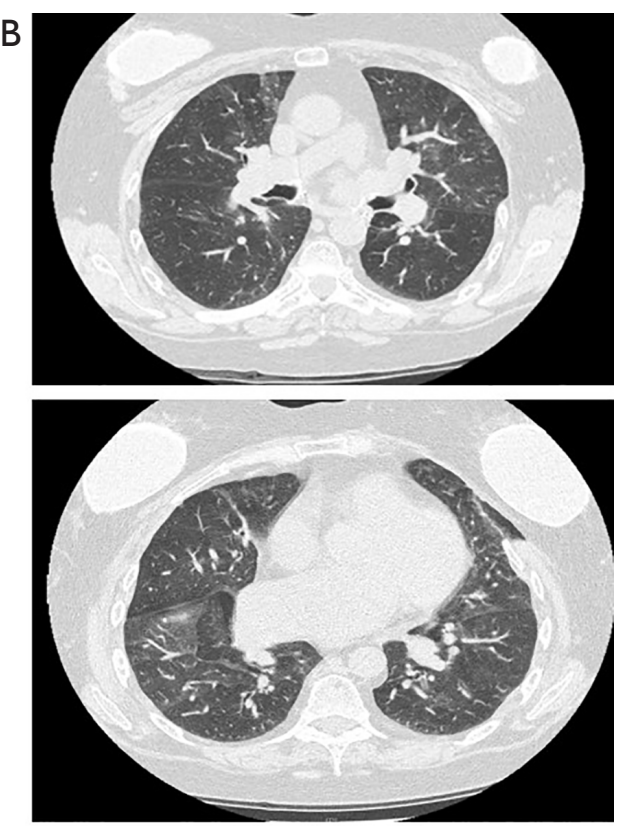

4b: BOS

Figure 4 Upper zone predominant fibrosis and reticulation seen in RAS (A) compared to the mosaic perfusion and bronchial wall thickening appearance in BOS (B). RAS, restrictive allograft syndrome; BOS, bronchiolitis obliterans syndrome.

as a result of gas trapping and hyperinflation $(15,23)$, the consensus document acknowledges that the diagnosis of a restrictive form of CLAD can be made if FVC declines from baseline and $\mathrm{FEV}_{1} / \mathrm{FVC}$ ratio is elevated (12) recognising the body of work highlighting the poor prognosis associated with FVC decline (16-20).

\section{Mixed and undefined phenotypes of CLAD}

Despite the extensive evolution of the description and characterisation of CLAD there remains categories of lung function decline and radiological changes that are not clearly defined. Whilst many lung transplant recipients with CLAD demonstrate a predominantly obstructive or restrictive ventilatory pattern of dysfunction, others exist who present with a mixed ventilatory defect or one that evolves from one pattern to another over time $(14,16,17)$. In addition, others present with obstructive changes on lung function that could be consistent with BOS but have evidence of opacities on chest imaging and others present with restrictive ventilatory defects on lung function and no changes on imaging (6). These patterns remain as yet undefined phenotypes of CLAD, potentially representing early changes of BOS or RAS; or another entity yet to be characterised.

Evidence in multiple studies, in particular those including histopathological evaluation of lung transplant recipients with CLAD have shown that whilst there is often a predominant pathology present other changes are also often seen. The underlying pathological abnormality associated with BOS is accepted to be OB which begins as an excess of subepithelial fibrosis tissue and progresses to obliteration of the lumen of the bronchiole by fibrosis. The pathology of RAS on the other hand has demonstrated diffuse alveolar damage, parenchymal fibrosis and fibroelastosis with or without pleural involvement $(13,24-26)$. A multitude of studies have also shown that $\mathrm{OB}$ of various stages coexists with the fibrotic changes of RAS. Yousem et al., first identified the extensive OB lesions seen in five pathological specimens of heart-lung transplant recipients (27). Whilst they identified $\mathrm{OB}$ as the major pathological process and predicted its influence on long-term post-transplant outcomes, they also identified interstitial and pleural fibrosis in some of their specimens. A later study by Martinu et al. examining the explants of 12 individuals undergoing retransplantation for BOS, found that in addition to changes of $\mathrm{OB}, 16 \%$ had evidence of extensive pleural and parenchymal fibrosis (28). The coexistence of the changes 
of both OB with features consistent with RAS, has been supported by more recent histopathological studies which have reported a prevalence of $\mathrm{OB}$ in pathological specimens of transplant recipients diagnosed with RAS of $62-100 \%$ $(24,25,29)$. This identification of multiple pathological features may explain the mixed ventilatory patterns of lung function seen in those with CLAD and the yet as undefined phenotypes specified by the consensus document.

\section{Areas of further study}

The aim of LTx remains the improvement in both the survival and quality of life of individuals with end-stage lung disease. Though normalisation of lung function, elimination of breathlessness and the return to optimal respiratory function for the individual remains the stated goal, little research has focused on what "normal" lung function following transplant represents. A recent paper by Liu et al. has illustrated that failure to normalise spirometry at baseline based on population predicted values is an independent predictor for mortality following LTx (20). This has been termed baseline lung allograft dysfunction (BLAD). Though not strictly part of the definition of CLAD, this failure to normalize lung function could represent a failure to attain potential and may provide evidence of early allograft injury or dysfunction that affects long-term outcomes. It may be that normal population predicted values for lung function can add further insight into the post-transplant progress of lung transplant recipients and that the attainment of peak lung function, rather than just loss of lung function and the development of CLAD could further inform our understanding of the early influences and circumstances that influence long-term outcomes.

The incorporation of imaging within the definitions of CLAD highlights the value of multimodal diagnostic tools in the classification of these complex phenotypes. As imaging techniques improve and machine-learning becomes a tool more readily accessible, it is possible that improved accuracy in diagnosis can be achieved. A number of recent studies have utilised machine learning to interrogate CT imaging in lung transplant recipients and whilst they remain in early stages of investigation there is promise that differences in lung density and deformation seen on HRCT may lead to early identification of individuals with RAS (30-32).

With regard to CLAD, another major area requiring exploration is the role of antibody mediated rejection
(AMR). There are definite clues that chronic allograft damage, as seen histologically in association with the apical fibrotic changes on HRCT used to characterize RAS, are linked to antibody-associated disease $(33,34)$. While imaging has become an increasingly important diagnostic tool in classifying CLAD phenotypes, histology from transbronchial biopsies is variable in its utility to assist with diagnosis and phenotyping. This has been demonstrated back the lack of specific reproducible features on histology in AMR (10). While this is partly due to suboptimal sampling, interobserver agreement is inconsistent and represents the subjective element of histopathology reporting. Molecular assessment using a microarraybased diagnostic system has been trialled as an alternative to histological diagnosis with early promising results in lung and other solid organ transplants (35) in detecting a molecular signal of different CLAD phenotypes that is highly reproducible and requires less lung parenchyma than traditional histology methods.

\section{The need to revise our standard classifiers for causes of death}

With the evolution of the definition of allograft failure from BOS to CLAD comes a need to review registry classification and nomenclature regarding the impact of allograft failure on survival. ISHLT and Organ Procurement and Transplantation Network (OPTN)/ Scientific Registry of Transplant Recipients (SRTR) are the largest registries incorporating LTx outcome data but information regarding causes of death (COD) is limited and utilises classifications that are not contemporary with the evolved definitions $(2,36)$. The differences in the clinical and research definitions of CLAD compared to how registries collate and re-stratify survival data has significant implications for the accuracy of registry reporting. Further analysis of this large dataset is therefore more challenging. This gap needs to be bridged with an update in COD definitions.

Levin et al. (37) has proposed an alternative classification scheme for COD. Rather than using a single descriptor for COD, they suggest a more nuanced approach, incorporating the strength of association with COD for both CLAD and infection. Not only does this allow the incorporation of updated CLAD classifications when describing COD (6) but also assigns a score to determine whether CLAD was the primary COD, a major contributor to COD, present but not associated with COD or not present at all. This 
draws parallels from other solid organ and bone marrow transplantation registries, where death is reported as "with or without a functioning graft" and "relapse-related or non-relapse related", respectively $(38,39)$. Their proposed scheme also includes an "Other" category, which accounts for non-infection and non-CLAD related deaths. As the authors of this paper suggest, improving COD reporting and data collection will enhance our understanding of changing practices and attitudes towards the dynamic patterns of CLAD and infection following LTx.

\section{Conclusions}

The definition of CLAD remains dynamic and reflects the development of greater understanding of its evolution from the initial identification of BOS to the different CLAD phenotypes so far identified. Despite their limitations, the CLAD consensus definitions go a long way in developing standardised methods for describing CLAD phenotypes and aim to bring consensus to a field that has evolved significantly over the last decade. With these new definitions of CLAD, further studies focussing in particular on longitudinal changes in lung function, prevalence, risk factors and prognostic variables for the different phenotypes of CLAD along with radiological and pathological sophistication in CLAD diagnosis will hopefully lead not only to clearer definitions but also therapeutic interventions to improve outcomes.

\section{Acknowledgments}

Funding: None.

\section{Footnote}

Provenance and Peer Review: This article was commissioned by the Guest Editors (Masaaki Sato and Dong Tian) for the series "Strategies to Achieve Long-Term Success of Lung Transplantation" published in Annals of Translational Medicine. The article was sent for external peer review organized by the Guest Editors and the editorial office.

Conflicts of Interest: The series "Strategies to Achieve LongTerm Success of Lung Transplantation" was commissioned by the editorial office without any funding or sponsorship. The authors have no other conflicts of interest to declare.

Ethical Statement: The authors are accountable for all aspects of the work in ensuring that questions related to the accuracy or integrity of any part of the work are appropriately investigated and resolved.

Open Access Statement: This is an Open Access article distributed in accordance with the Creative Commons Attribution-NonCommercial-NoDerivs 4.0 International License (CC BY-NC-ND 4.0), which permits the noncommercial replication and distribution of the article with the strict proviso that no changes or edits are made and the original work is properly cited (including links to both the formal publication through the relevant DOI and the license). See: https://creativecommons.org/licenses/by-nc-nd/4.0/.

\section{References}

1. Paraskeva MA, Levin KC, Westall GP, et al. Lung transplantation in Australia, 1986-2018: more than 30 years in the making. Med J Aust 2018;208:445-50.

2. Chambers DC, Cherikh WS, Golfarb SB, et al. The International Thoracic Organ Transplant Registry of the International Society for Heart and Lung Transplantation: Thirty-fifth Adult lung and heart-lung transplant report-2018; Focus theme: Multiorgan Transplantation. J Heart Lung Transplant 2018;37:1169-83.

3. Burke CM, Theodore J, Dawkins KD, et al. Posttransplant obliterative bronchiolitis and other late lung sequelae in human heart-lung transplantation. Chest 1984;86:824-9.

4. Cooper JD, Billingham M, Egan T, et al. A working formulation for the standardization of nomenclature and for clinical staging of chronic dysfunction in lung allografts. International Society for Heart and Lung Transplantation. J Heart Lung Transplant 1993;12:713-6.

5. Meyer KC, Raghu G, Verleden GM, et al. An international ISHLT/ATS/ERS clinical practice guideline: diagnosis and management of bronchiolitis obliterans syndrome. Eur Respir J 2014;44:1479-503.

6. Verleden GM, Glanville AR, Lease ED, et al. Chronic lung allograft dysfunction: Definition, diagnostic criteria, and approaches to treatment-A consensus report from the Pulmonary Council of the ISHLT. J Heart Lung Transplant 2019;38:493-503.

7. Vanaudenaerde BM, Meyts I, Vos R, et al. A dichotomy in bronchiolitis obliterans syndrome after lung transplantation revealed by azithromycin therapy. Eur Respir J 2008;32:832-43.

8. Pakhale SS, Hadjiliadis D, Howell DN, et al. Upper lobe 
fibrosis: a novel manifestation of chronic lung allograft dysfunction in lung transplantation. J Heart Lung Transplant 2005;24:1260-8.

9. Sato $M$, Waddell TK, Wagnetz $U$, et al. Restrictive allograft syndrome (RAS): a novel form of chronic lung allograft dysfunction. J Heart Lung Transplant 2011;30:735-42.

10. Levine DJ, Glanville AR, Aboyoun C, et al. Antibodymediated rejection of the lung: A consensus report of the International Society for Heart and Lung Transplantation. J Heart Lung Transplant 2016;35:397-406.

11. Walton DC, Hiho SJ, Cantwell LS, et al. HLA Matching at the eplet level protects against chronic lung allograft dysfunction. Am J Transplant 2016;16:2695-703.

12. Glanville AR, Verleden GM, Todd JL, et al. Chronic lung allograft dysfunction: Definition and update of restrictive allograft syndrome-A consensus report from the Pulmonary Council of the ISHLT. J Heart Lung Transplant 2019;38:483-92.

13. Sato M, Hwang DM, Ohmori-Matsuda K, et al. Revisiting the pathologic finding of diffuse alveolar damage after lung transplantation. J Heart Lung Transplant 2012;31:354-63.

14. Sato M, Hwang DM, Waddell TK, et al. Progression pattern of restrictive allograft syndrome after lung transplantation. J Heart Lung Transplant 2013;32:23-30.

15. Kneidinger N, Milger K, Janitza S, et al. Lung volumes predict survival in patients with chronic lung allograft dysfunction. Eur Respir J 2017;49:1601315.

16. Verleden GM, Vos R, Verleden SE, et al. Survival determinants in lung transplant patients with chronic allograft dysfunction. Transplantation 2011;92:703-8.

17. Suhling H, Dettmer S, Greer M, et al. Phenotyping Chronic Lung Allograft Dysfunction Using Body Plethysmography and Computed Tomography. Am J Transplant 2016;16:3163-70.

18. Belloli EA, Wang X, Murray S, et al. Longitudinal Forced Vital Capacity Monitoring as a Prognostic Adjunct after Lung Transplantation. Am J Respir Crit Care Med 2015;192:209-18.

19. Todd JL, Jain R, Pavlisko EN, et al. Impact of forced vital capacity loss on survival after the onset of chronic lung allograft dysfunction. Am J Respir Crit Care Med 2014;189:159-66.

20. Liu J, Jackson K, Weinkauf J, et al. Baseline lung allograft dysfunction is associated with impaired survival after double-lung transplantation. J Heart Lung Transplant
2018;37:895-902.

21. American Thoracic Society. Lung function testing: selection of reference values and interpretative strategies. Am Rev Respir Dis 1991;144:1202-18.

22. DerHovanessian A, Todd JL, Zhang A, et al. Validation and refinement of chronic lung allograft dysfunction phenotypes in bilateral and single lung recipients. Ann Am Thorac Soc 2016;13:627-35.

23. Suhling H, de Wall C, Rademacher J, et al. Low exercise tolerance correlates with reduced inspiratory capacity and respiratory muscle function in recipients with advanced chronic lung allograft dysfunction. Transplantation 2013;95:1045-50.

24. Jonigk D, Rath B, Borchert P, et al. Comparative analysis of morphological and molecular motifs in bronchiolitis obliterans and alveolar fibroelastosis after lung and stem cell transplantation. J Pathol Clin Res 2016;3:17-28.

25. Ofek E, Sato M, Saito T, et al. Restrictive allograft syndrome post lung transplantation is characterized by pleuroparenchymal fibroelastosis. Mod Pathol 2013;26:350-6.

26. von der Thüsen JH, Vandermeulen E, Vos R, et al. The histomorphological spectrum of restrictive chronic lung allograft dysfunction and implications for prognosis. Mod Pathol 2018;31:780-90.

27. Yousem SA, Burke CM, Billingham ME. Pathologic pulmonary alterations in long-term human heart-lung transplantation. Hum Pathol 1985;16:911-23.

28. Martinu T, Howell DN, Davis RD, et al. Pathologic correlates of bronchiolitis obliterans syndrome in pulmonary retransplant recipients. Chest 2006;129:1016-23.

29. Montero MA, Osadolor T, Khiroya R, et al. Restrictive allograft syndrome and idiopathic pleuroparenchymal fibroelastosis: do they really have the same histology? Histopathology 2017;70:1107-13.

30. Horie M, Levy L, Houbois C, et al. Lung Density Analysis Using Quantitative Chest CT for Early Prediction of Chronic Lung Allograft Dysfunction. Transplantation 2019. [Epub ahead of print].

31. Horie M, Salazar P, Saito T, et al. Quantitative chest CT for subtyping chronic lung allograft dysfunction and its association with survival. Clinical Transplantation 2018;32:e13233.

32. Saito T, Horie M, Sato M, Net al. Low-dose computed tomography volumetry for subtyping chronic lung allograft dysfunction. J Heart Lung Transplant 2016;35:59-66. 
33. Roux A, Bendib Le Lan I, Holifanjaniana S, et al. Antibody-mediated rejection in lung transplantation: clinical outcomes and donor-specific antibody characteristics. Am J Transplant 2016;16:1216-28.

34. Verleden SE, Vanaudenaerde BM, Emonds MP, et al. Donor-specific and -non specific HLA antibodies and outcome post lung transplantation. Eur Respir J 2017;50:1701248.

35. Halloran KM, Parkes MD, Chang J, et al. Molecular assessment of rejection and jury in lung transplant biopsies. J Heart Lung Transplant 2019;38:504-13.

36. Valapour M, Lehr CJ, Skeans MA, et al. OPTN/SRTR 2016 Annual Data Report: Lung. Am J Transplant 2018;18

Cite this article as: Kotecha S, Paraskeva MA, Levin K, Snell GI. An update on chronic lung allograft dysfunction. Ann Transl Med 2020;8(6):417. doi: 10.21037/atm.2020.01.05
Suppl 1:363-433.

37. Levin K, Levvey BJ, Paraskeva M, et al. Breathing life into lung transplant mortality definitions and reporting. J Heart Lung Transplant 2019. [Epub ahead of print].

38. Hart A, Smith JM, Skeans MA, et al. OPTN/SRTR 2016 Annual Data Report: Kidney. Am J Transplant 2018;18 Suppl 1:18-113.

39. Wadhwa A, Chen Y, Holmqvist A, et al. Late Mortality after Allogeneic Blood or Marrow Transplantation for Inborn Errors of Metabolism: A Report from the Blood or Marrow Transplant Survivor Study-2 (BMTSS-2). Biol Blood Marrow Transplant 2019;25:328-34. 